\title{
Interaural Time Difference Processing in the Mammalian Medial Superior Olive: The Role of Glycinergic Inhibition
}

\author{
Michael Pecka, ${ }^{1,3}$ Antje Brand, ${ }^{2}$ Oliver Behrend, ${ }^{2}$ and Benedikt Grothe ${ }^{1,2,3}$ \\ ${ }^{1}$ Division of Neurobiology, Department Biology II, Ludwig-Maximilians University Munich, D-82152 Martinsried, Germany, ${ }^{2}$ Max Plank Institute of \\ Neurobiology, D-82152 Martinsried, Germany, and 3Bernstein Center for Computational Neuroscience, D-81377 Munich, Germany
}

The dominant cue for localization of low-frequency sounds are microsecond differences in the time-of-arrival of sounds at the two ears [interaural time difference (ITD)]. In mammals, ITD sensitivity is established in the medial superior olive (MSO) by coincidence detection of excitatory inputs from both ears. Hence the relative delay of the binaural inputs is crucial for adjusting ITD sensitivity in MSO cells. How these delays are constructed is, however, still unknown. Specifically, the question of whether inhibitory inputs are involved in timing the net excitation in MSO cells, and if so how, is controversial. These inhibitory inputs derive from the nuclei of the trapezoid body, which have physiological and structural specializations for high-fidelity temporal transmission, raising the possibility that well timed inhibition is involved in tuning ITD sensitivity. Here, we present physiological and pharmacological data from in vivo extracellular MSO recordings in anesthetized gerbils. Reversible blockade of synaptic inhibition by iontophoretic application of the glycine antagonist strychnine increased firing rates and significantly shifted ITD sensitivity of MSO neurons. This indicates that glycinergic inhibition plays a major role in tuning the delays of binaural excitation. We also tonically applied glycine, which lowered firing rates but also shifted ITD sensitivity in a way analogous to strychnine. Hence tonic glycine application experimentally decoupled the effect of inhibition from the timing of its inputs. We conclude that, for proper ITD processing, not only is inhibition necessary, but it must also be precisely timed.

Key words: sound localization; pharmacology; strychnine; population coding; superior oliviary complex; brainstem

\section{Introduction}

Auditory space is synthesized by our brain based on onedimensional movements of the two tympanic eardrums. Spectral cues and differences in level or arrival time of a sound at the two ears are used by the brain for performing this computational task. Interaural time differences (ITDs) are the dominant cue for localizing low-frequency sounds, which are most important for larger mammals (including humans) and small mammals living in habitats that require detection of distant sounds and longrange communication (like Mongolian gerbils). These species have well developed low-frequency hearing and a well developed medial superior olive (MSO) with principal neurons highly sensitive to ITDs (Goldberg and Brown, 1969; Moushegian et al., 1975; Crow et al., 1978; Yin and Chan, 1990; Spitzer and Semple, 1995).

Until recently, considerations of mammalian ITD processing

Received Sept. 10, 2007; revised May 13, 2008; accepted May 23, 2008.

This work was supported by The Max Planck Society (B.G.), the German Research Foundation (Deutsche Forschungsgemeinschaft) (Gr1205/12-1; GR1205/14-1, GRK 1091), and Bundesministerium für Bildung und Forschung (Project 3.5 of the Bernstein Center for Computational Neuroscience). We thank Drs. D. H. Sanes, N. Lesica, D. McAlpine, and G. D. Pollak for helpful discussions and critical comments on this manuscript. We are thankful to Dr. R. M. Burger for technical advice. C. Schulte provided excellent assistance with the histology.

Correspondence should be addressed to Prof. Dr. Benedikt Grothe, Department Biology II, Biocenter, LudwigMaximilians University Munich, Grosshaderner Strasse2,D-82152 Martinsried, Germany.E-mail:neurobio@Imu.de.

A. Brand's present address: Helmholtz Zentrum München-Deutsches Forschungszentrum für Gesundheit und Umwelt, GmbH, D-85764 Neuherberg, Germany.

0 . Behrend's present address: Munich Center for Neurosciences-Brain and Mind, D-82152 Martinsried, Germany. D0I:10.1523/JNEUROSCI.1660-08.2008

Copyright $\odot 2008$ Society for Neuroscience $\quad$ 0270-6474/08/286914-12\$15.00/0 were dominated by a model proposed by Jeffress in 1948. This model assumes coincidence detection of binaural excitatory inputs with systematically varying axonal conduction time: Neurons respond maximally to ITDs that compensate for the differences in axonal conduction time of the excitatory inputs from both ears. The systematic arrangement of axons with different conduction times tunes neurons to different ITDs, creating a place code whereby the location of peak activity forms a map of auditory space. This arrangement has been verified both structurally and physiologically for the bird ITD detection system (for review, see Carr and Soares, 2002; Grothe et al., 2004). Mammals, however, developed ITD processing independently (Manley et al., 2004) and the structural and functional mechanisms of ITD processing in mammals are not as well understood. Although findings of anatomical studies in the cat seemed to be consistent with a delay line organization for the contralateral excitatory MSO inputs (Smith et al., 1993; Beckius et al., 1999), a number of recent studies indicate other or additional mechanisms underlying ITD tuning of MSO neurons and/or suggest a neuronal representation of ITDs that is different to the place coding in the Jeffress model (for review, see Grothe, 2003; Palmer, 2004). Particularly controversial is the role of synaptic inhibition in tuning MSO neurons to specific ITDs (Joris and Yin, 2007). There is anatomical (Clark, 1969; Perkins, 1973; Wenthold et al., 1987; Cant, 1991; Cant and Hyson, 1992; Kuwabara and Zook, 1992) and physiological evidence (Grothe and Sanes, 1993, 1994; Grothe and Park, 1998) for strong glycinergic inputs onto MSO principal neurons. These inputs derive from the medial nucleus of the 
trapezoid body (MNTB) and, to a lesser extent (Grothe and Sanes, 1993), the lateral nucleus of the trapezoid body (LNTB). The MNTB in particular (von Gersdorff and Borst, 2002), but also the LNTB (Spirou et al., 1998), show pronounced specializations for high-fidelity temporal transmission. Moreover, the glycinergic MSO inputs are restricted to the cell somata (Clark, 1969; Perkins, 1973; Kapfer et al., 2002), and this seems to be crucial for shaping the range of ITDs to which MSO neurons are tuned (Seidl and Grothe, 2005). The range of ITDs that neurons are sensitive to has led to a second controversy about the strategy of encoding location: is it the peak of the ITD function that encodes a location, and thus the place of maximal activity that shifts with location as proposed by Jeffress, or is it the slope of the function, in which firing rates change markedly with location, and thus the coding is by rate rather than place?

Here, we recorded from MSO neurons in the gerbil and present evidence in support of a rate code and for a strong influence of glycinergic inhibition on ITD tuning of MSO neurons to stimulus onset as well as to ongoing activity. We argue that these effects cannot be explained by tonic but only by precisely timed inhibition. Part of this data were published previously as a short communication (Brand et al., 2002).

\section{Materials and Methods}

Animals and surgery. Experiments were approved by the German animal welfare law (209.1/211-2531-40/01 and 55.2-1-54-2531-57-05). Adult Mongolian gerbils (Meriones unguiculatus), weighing between 60 and $100 \mathrm{~g}$, were initially anesthetized with an intraperitoneal injection $(0.5$ $\mathrm{ml} / 100 \mathrm{~g}$ body weight) of a mixture of ketamine (20\%) and xylazine (2\%) diluted in $0.9 \% \mathrm{NaCl}$ solution. During surgery, analgesia was reinforced by administration of additional doses of anesthesia. An adequate analgesic state of the animal was ascertained regularly throughout the experiment by testing for absence of motor reflexes and monitoring breathing rate. To secure a constant state of anesthesia during electrophysiological recordings, supplementary doses of $0.05-0.1 \mathrm{ml}$ of the same mixture were given subcutaneously every $30 \mathrm{~min}$ or when needed. The animal was transferred to a sound-attenuated chamber and mounted in a custommade stereotaxic instrument (Schuller et al., 1986). Constant body temperature $\left(37-39^{\circ} \mathrm{C}\right)$ was maintained using a thermostatically controlled heating blanket. A craniotomy $(\sim 2.5 \times 2 \mathrm{~mm})$ was performed just lateral to midline of the skull and caudal to the posterior aspect of the transverse sinus. The dura was opened and the underlying cerebellum partially aspirated to expose the floor of the fourth ventricle. For a subset of experiments (pharmacology with strychnine), access to the brain was obtained by an opening of the dura at the foramen magnum. Ringer's solution was frequently applied to the opening to prevent dehydration of the brain. After recordings (10-15 h) and a lethal injection of anesthesia, the animals were perfused intracardially with heparinized $\mathrm{NaCl}$ solution $(0.9 \%)$ followed by $4 \%$ paraformaldehyde (PFA) solution. Afterward, the brain was retained in 4\% PFA for histological processing.

Acoustic stimuli and sound delivery. Acoustic stimuli were digitally generated at a sampling rate of $50 \mathrm{kHz}$ by TDT System II or III (Tucker-Davis Technologies), converted to analog signals (DA3-2/RP2-1; Tucker-Davis Technologies), attenuated to desired levels (PA4/PA5; Tucker-Davis Technologies), and delivered to the earphones (ES1; Tucker-Davis Technologies/TD990; Beyer Dynamics/Sony; Stereo Dynamic Earphones; MDR-EX70LP). For details and calibration procedures, see Siveke et al. (2006). Stimuli had $\cos ^{2}$-function gated rise-fall times of $5 \mathrm{~ms}$ that were added to given stimulus durations and were presented with equal intensities at the two ears. To search for acoustic responses, 100 or $200 \mathrm{~ms}$ uncorrelated noise bursts were delivered binaurally. When a neuron was encountered, its characteristic frequency $(\mathrm{CF})$ and threshold were determined. The frequency that elicited responses at the lowest sound intensity was defined as CF; the lowest sound intensity evoking a noticeable response at $\mathrm{CF}$ was defined as threshold of the neuron. These properties were determined by a frequency-versus-level stimulus matrix using 200 $\mathrm{ms}$ pure tones. All stimuli were presented in a randomized, interleaved order. CFs ranged from 165 to $4800 \mathrm{~Hz}$ with thresholds between 0 and 65 $\mathrm{dB}$ sound pressure level (SPL). In addition to binaural presentation, for the majority of neurons the same stimulus matrix was presented monaurally so that the excitatory or inhibitory nature of the inputs from both ears could be determined. Most low-frequency neurons were then tested for ITD sensitivity first by presenting an ITD-versus-intensity stimulus matrix (duration, $100 \mathrm{~ms}$ ) at $\mathrm{CF}$ (or below for CFs $>1300 \mathrm{~Hz}$ ). The intensity at which the neuron showed best modulation in response to different ITDs was determined (typically $20-30 \mathrm{~dB}$ above threshold). This intensity was subsequently applied for all additional stimulations. ITD-versus-frequency stimulus matrices were presented with either 50 or $200 \mathrm{~ms}$ long pure tones. Five or nine frequencies centered on CF were tested over a range of ITDs equivalent to at least two cycles of the stimulation frequency and 10 interaural phase differences (IPDs) per period of the $\mathrm{CF}$ of a neuron. The frequency that elicited the highest response at the central peak of the ITD function was defined as best frequency (BF). During experiments involving iontophoretic application of glycine, ITDs were presented at BF with $50 \mathrm{~ms}$ stimulus duration and 10 ITDs per period. During iontophoretic application of strychnine, which were conducted before a modification of the setup for reasons not related to this study, $100 \mathrm{~ms}$ long pure tones (including $5 \mathrm{~ms}$ rise and $5 \mathrm{~ms}$ fall time) were presented with ITDs of -1 to +1 ms with 0.1 or $0.2 \mathrm{~ms}$ increments. In two cells, ITD functions were obtained using trains of narrowband pulses (duration, $5 \mathrm{~ms}$; center frequency, BF; five pulses per sweep).

Electrophysiological recordings and data analysis. Action potentials from single neurons were recorded extracellularly using glass electrodes (impedance, 5-20 M $\Omega$ ) filled with $1 \mathrm{M} \mathrm{NaCl}$ or $\% \mathrm{HRP}$ (Sigma-Aldrich) diluted in $10 \% \mathrm{NaCl}$. For the dorsal approach, the recording electrode was tilted caudally by 18 or $20^{\circ}$, whereas for the approach through the foramen magnum the animals head was tilted by $60^{\circ}$. Electrodes were advanced under remote control, using a motorized micromanipulator (Digimatic) and a piezodrive [PM-10-1 (World Precision Instruments) or Inchworm controller 8200 (EXFO Burleigh Products Group)]. Recordings were amplified, filtered, and A/D-converted (RP2-1; TuckerDavis Technologies), and the digitized signals were fed to the computer. Clear isolation of action potentials from single neurons was guaranteed by visual inspection on a spike-triggered oscilloscope (to assure a stable shape and amplitude of the action potential) and by off-line spike cluster analysis (Brainware; Tucker-Davis Technologies).

Responses to ITD stimuli were analyzed for best IPD and vector strength (VS) for each frequency using vector analysis (Goldberg and Brown, 1969; Yin and Kuwada, 1983). Significant best IPDs were scored based on the $p<0.001$ significance level of the Rayleigh test of uniformity (Mardia, 1972). Significant best IPDs were plotted against stimulation frequency, and the linear regression of these values was calculated (at least three significant frequencies). Thereby the characteristic delay (CD) and the characteristic phase $(\mathrm{CP})$ of the neuron were obtained (Yin and Kuwada, 1983). The CD value denotes the estimated ITD at which the ITD function showed constant relative amplitude with different frequencies. The $\mathrm{CP}$ value describes the mean interaural phase at which the $\mathrm{CD}$ occurred. A CP value near or at 0 cycles indicates that the CD occurred near or at the peak of an ITD function and is indicative of a coincidence of binaural excitation (peak-type). Conversely, CP values near 0.5 cycles indicate $\mathrm{CD}$ at the trough of an ITD function, which can be explained by a coincidence of excitatory and inhibitory inputs (trough-type). We calculated CD and CP both with a weighted regression as described by Kuwada et al. (1987) or Spitzer and Semple (1995) and with a nonweighted regression. The two ways of calculation yielded similar results for both $\mathrm{CD}$ and $\mathrm{CP}$ in all neurons. Thus, we only present $\mathrm{CD}$ and $\mathrm{CP}$ values calculated without weighting. The best ITD, the most favorable ITD of a neuron, was obtained by multiplying the best IPD with the period of the stimulation frequency. The ITD at which the ITD function of a neuron had its steepest slope was calculated by fitting a Gaussian function with an additional offset parameter to the central period of the ITD function at BF (the central period was defined as one cycle of IPDs centered on best IPD). The average $R^{2}$ value of the fit was $0.94 \pm 0.06$ (SD). For pharmacological experiments, the significance of changes in the distribution of spikes over the measured ITDs within the central period of the ITD function was assessed using the $p<0.05$ criterion of 
Watson's $U^{2}$ test (software, Oriana 2; Kovach Computing Services). Population statistics on differences in normalized response rates were computed by normalizing ITD or IPD functions of control and drug condition of each neuron and calculating the changes in normalized response rates between control and drug condition at three ITDs (for strychnine) or IPDs (for glycine) to the left-hand side as well as to the right-hand side of the best ITD/IPD of each cell under control conditions. Subsequently, significance of the changes in the population of cells was assessed separately for left- and right-hand side and for each drug condition using the Student $t$ test.

For analysis of the onset component of responses, only the first $10 \mathrm{~ms}$ of the stimulus-related responses were considered. For ongoing components, the first $10 \mathrm{~ms}$ of the stimulus-related response were discarded, if not stated otherwise. CD, CP, and best IPD of each frequency were determined in the way described above. Neurons were included in the analysis only if CD and CP could be calculated for both onset and ongoing component. Analysis of "first spike" onset component was only performed on responses of neurons for which first spikes in response to all repetitions presented could be binned into one $2 \mathrm{~ms}$ time window.

Iontophoresis and drugs. To mark a recording site after successful recordings, HRP (Sigma-Aldrich) was ejected through the recording pipette using a current source (HV-TR150; NPI) connected to the recording pipette. A current of $+1 \mu \mathrm{A}$ was applied to the pipette for 30-50 s.

Five-barrel glass pipettes with tip size of $15-30 \mu \mathrm{m}$ were used for drug iontophoresis. Barrels were backfilled with glycine (amino-ethanoic acid, pH 3.5-4; 100 mм; Sigma-Aldrich); strychnine, pH 3.5 (10 mм); or $\mathrm{NaCl}, \mathrm{pH} 3(1 \mathrm{~m})$. The center barrel was filled with either $2 \mathrm{~m}$ sodium acetate or $1 \mathrm{M} \mathrm{NaCl}$ for current balancing. Retention and ejection currents were applied via a Neurophore $\mathrm{BH}-2$ system and IP-2 current pumps (Harvard Apparatus). Strychnine was retained with a current of $-24 \mathrm{nA}$ and ejected with a current of $\leq 240 \mathrm{nA}$. Glycine was retained in barrels with a current of $-20 \mathrm{nA}$ and ejected with a current of typically +20 to $+200 \mathrm{nA}$.

Histology. Brains were sectioned frontally ( $40 \mu \mathrm{m}$ thickness), and sections were stained for HRP with diaminobenzidine, intensified by cytochrome (Adams, 1977). After mounting to a glass slide, the sections were counterstained by standard protocol using Neutral Red. Sections were analyzed by light microscopy, and recording sites were captured digitally. In some cases, no distinct HRP staining was found but recording sites could be reconstructed from the track of the recording electrode that was clearly visible because of either diffuse HRP staining or diaminobenzidine-stained erythrocytes caused by lesions induced by the electrode (see Fig. $1 A$, black arrow).

\section{Results}

Here, we report on single-cell recordings from 62 MSO neurons. Pure-tone ITD functions were recorded with neuronal BFs ranging from 200 to $1200 \mathrm{~Hz}$; note that CFs were occasionally much higher (see Materials and Methods). Recording sites for 46 of the 62 neurons were histologically confirmed or reconstructed to be within or directly adjacent to the MSO cell band (see Materials and Methods) (Fig. 1A). For 16 cells, no recording site could be determined, but these cells all showed clear evidence for binaural excitation via monaural stimulation from either ear and could be classified as peak type (for definition, see Materials and Methods). Moreover, the analysis of basic ITD sensitivity, which will be presented in the following section, revealed that the properties of cells recorded during histologically unconfirmed penetrations were indistinguishable from those of confirmed MSO recordings.

\section{Basic ITD properties}

We performed a detailed analysis of the sensitivity to ITDs in the fine structure of low-frequency pure tones in a subset of $48 \mathrm{MSO}$ neurons for which we were able to obtain recordings from a complete stimulus set (Fig. 1). Figure $1 B$ contains the distributions of CD and CP (for definitions, see Materials and Methods) among peak-type MSO neurons $(n=48)$. Both the CD and the
$\mathrm{CP}$ distribution were centered near zero with a slight asymmetry toward positive values. We also obtained data from 18 neurons with CPs $>0.25$, but these neurons were histologically determined not to be in the MSO or their recording sites could not be determined. Overall, the distributions are in line with previous data from the gerbil, rabbit, and cat MSO (Yin and Chan, 1990; Spitzer and Semple, 1995; Batra et al., 1997). When stimulated binaurally at CF and favorable ITDs, about one-third of the neurons (18 of 48) responded only to the beginning of a stimulus with a few action potentials independent of stimulus duration. The majority of neurons (30 of 48), however, responded in a primary-like sustained manner to favorable ITDs. Spiking of the neurons was highly synchronized to the fine structure of the tonal stimuli. The average vector strength at $\mathrm{BF}$ and best ITD was 0.46 $[ \pm 0.19$ (SD); $N=48$; average $\mathrm{BF}, 796 \mathrm{~Hz} \pm 213$ (SD); distribution of vector strengths as a function of $\mathrm{BF}$ is given in supplemental Fig. $1 A$ (available at www.jneurosci.org as supplemental material)]. Because the relative relevance of responses to the beginning of a stimulus for the localization of a sound source compared with ongoing responses is an issue of debate (Zurek, 1993; Akeroyd and Bernstein, 2001; Joris and Yin, 2007), we investigated whether ITD sensitivity of sustained responding neurons differed in the initial component ("onset"; first $10 \mathrm{~ms}$ ) from its ongoing component (remaining stimulus-related discharges). Figure $1 C 1-C 3$ shows ITD functions of one MSO neuron for five different frequencies between 200 and $300 \mathrm{~Hz}$ (BF of the neuron, $250 \mathrm{~Hz}$ ). In Figure 1C1, the total responses are plotted, whereas Figure 1, C2 and C3, shows the ITD functions separated into onset and ongoing component, respectively. Both onset and ongoing components exhibited strong modulation in response to different ITDs and had typical peak-type ITD sensitivity as expressed in the linear phase-frequency relationship intersecting the $y$-axis at IPD values close to 0 (Fig. 1C4). The CD for total, onset, and ongoing response of this neuron were 269,436 , and $283 \mu$ s, respectively, and the CPs were $0.13,0.03$, and 0.13 . Figure $1, D 1$ and $D 2$, shows the distributions of CD and CP for onset and ongoing response components in 21 cells that exhibited significant ITD sensitivity in their onset as well as sustained responses. Nine of the 30 sustained responding neurons were excluded from this analysis because of high variance in their onset latency $(n=$ 3 ) or insufficient number of spikes to calculate CD and CP in either the onset $(n=3)$ or ongoing $(n=3)$ component. The distribution of CPs and CDs was not significantly different for total, onset, or ongoing response $(p>0.49$ for all distributions, two-sided paired Student's $t$ test; $n=21$ ) (compare Fig. $1 B$ ). Moreover, plotting CD of onset against CD of ongoing component for each cell (Fig. 1E1) $(n=21)$ revealed no tendency in divergence, albeit values scattered slightly. The same observation holds true for the CPs of onset and ongoing components (Fig. $1 E 2)(n=21)$ and the VS of the two components (supplemental Fig. $1 B$, available at www.jneurosci.org as supplemental material). Most importantly, the preferred interaural phase differences (best IPDs) of each cell were similar for onset and ongoing component [correlation coefficient (cc), 0.8; $p<0.001$ ] (Fig. $1 F 1)(n=18)$. Furthermore, we analyzed the onset components in more detail by dividing them into the "first spike" (0-2 ms of response) and remaining $8 \mathrm{~ms}$ of the onset response. We compared the best IPDs derived from these subcomponents of the 10 ms onset response to the ongoing component. This analysis revealed that best IPDs derived from the $2-8 \mathrm{~ms}$ subcomponent of the onset response were very similar to ongoing best IPDs (cc, $0.89 ; p<0.001 ; n=20$ ) (Fig. 1F3). "First spike" onset best IPDs were less correlated to the ongoing best IPDs (cc, $0.49 ; p<0.1$; 

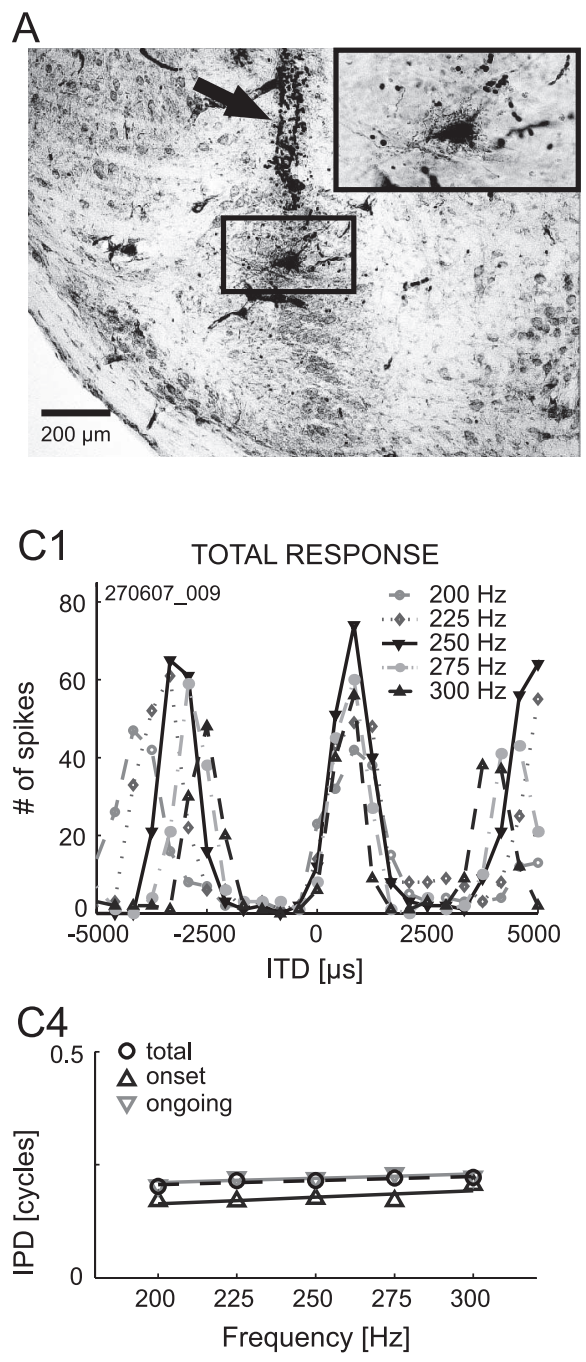

E1

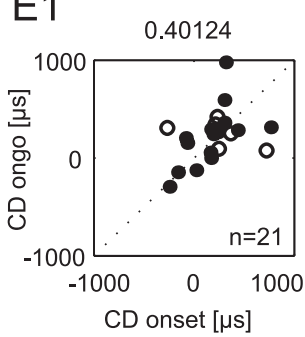

E2

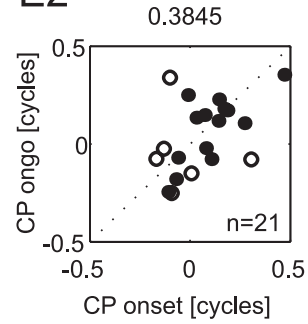

B1

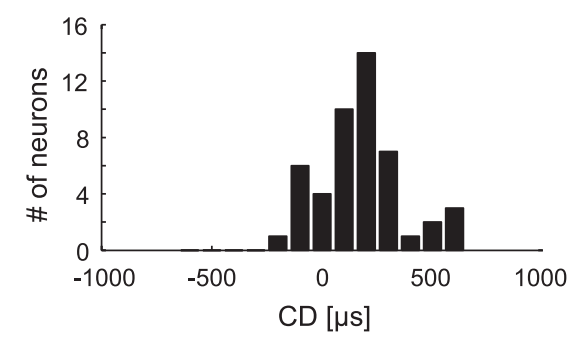

C2
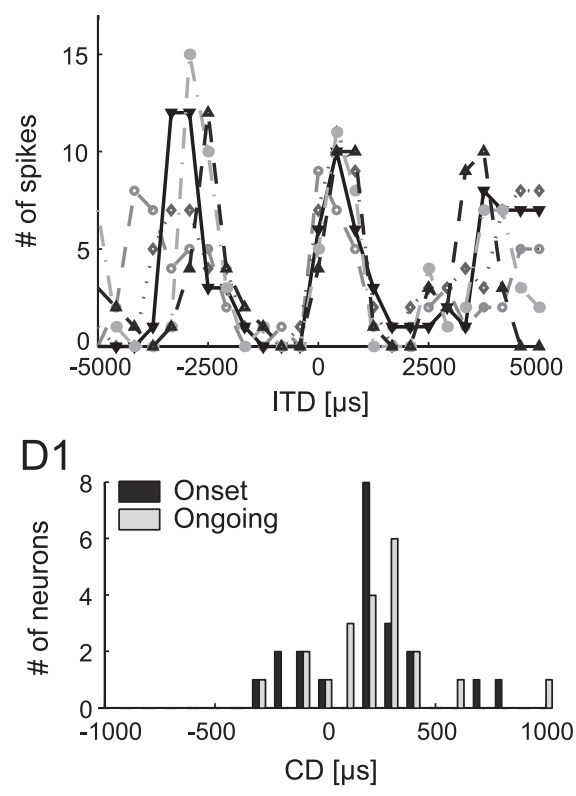

F1

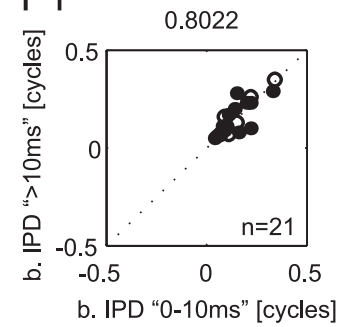

B2
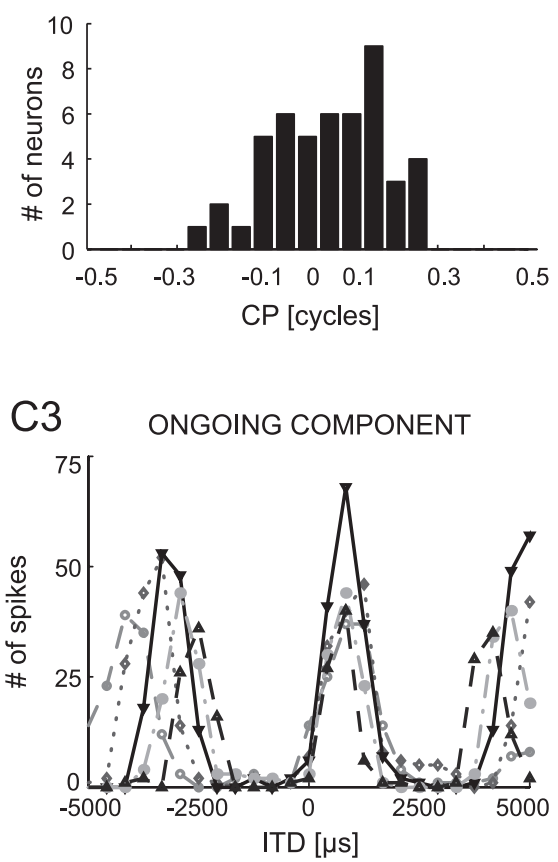

D2

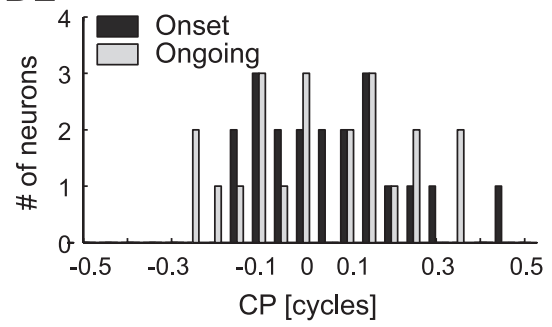

F3

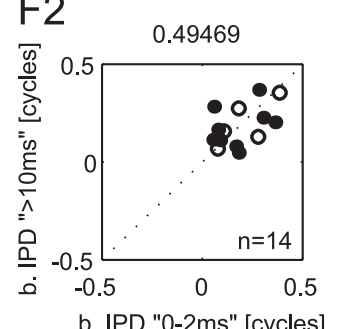

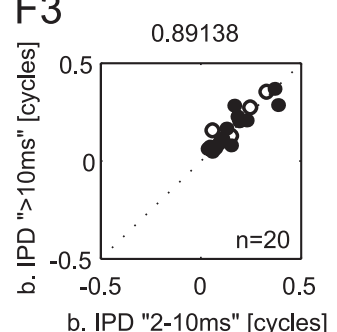

Figure 1. Basic properties of ITD sensitivity are equal for onset and ongoing responses. Stimulus durations were 50 or $200 \mathrm{~ms}$. A, Recording site for the example neuron shown in C. The MSO cell band and surrounding neuropil are clearly visible. HRP staining is present in the dorsal region of the MSO cell band. The electrode track of the multibarrel electrode is marked by the black arrow. The inset in top right corner shows higher magnification of the region demarked by the black square, with HRP staining being concentrated on the somatic area. $\boldsymbol{B}$, Distributions of CDs (B1) and CPs ( $\boldsymbol{B}$ ) of $48 \mathrm{MSO}$ neurons based on total responses. C1-C3, ITD functions of a low-frequency neuron ( $\mathrm{BF}, 250 \mathrm{~Hz}$ ) in response to pure tones ( 5 test frequencies; 8 repetitions; $50 \mathrm{~ms}$ duration). ITD functions are shown for total response (C1), onset component only (first $10 \mathrm{~ms}$ of total response) (C2), and ongoing component only (first $10 \mathrm{~ms}$ of total response excluded) (C3). (44, Phase-frequency plots for the ITD functions shown in C1-C3.D, Distributions of $C D(D 1)$ and $C P(D 2)$ for 21 of the 48 neurons in $B$, separately analyzed for onset and ongoing response components. $E$, Comparison between onset and ongoing response for $C D(E 1)$ and $C P(E 2)$. F, Best IPDs of first $10 \mathrm{~ms}$ of response were similar to best IPDs of ongoing responses (F1). Correlation was strongest if the first $2 \mathrm{~ms}$ of responses were excluded $(\boldsymbol{F} 3)$. Correlation coefficients are given on top of each panel. The open symbols indicate neurons with unconfirmed recording sites.

$n=14$ ) (Fig. $1 F)$; however, this result is most likely attributable to the low spike number for this subcomponent (typically $<20$ spikes).

Next, we examined the representation of the peak of the ITD functions within our population of MSO neurons and subsequently related it to the frequency tuning of the neurons, hence their BF, for 56 of the 62 MSO neurons (for the remaining 6 cells, no BF but only the CF was determined). We found a large pref- erence for ITD functions to peak at contralateral leading ITDs (55 of 56), a finding that is consistent with previous reports from the MSO (Yin and Chan, 1990; Spitzer and Semple, 1995; Batra et al., 1997). Normalizing best ITDs to the BFs of the neurons, the mean best IPD was 0.17 cycles \pm 0.08 (SD). Only one cell had an ipsilateral leading best ITD, which, however, was very close to 0 ITD $(-8 \mu \mathrm{s})$. The best ITD of most neurons was outside the "physiological range" (ITDs that naturally occur for single sound 
sources without interference of echoes or other stimuli; for the gerbil with its small interear distance, approximately $\pm 135 \mu \mathrm{s}$ ) (Maki and Furukawa, 2005), because only 10 of the 56 cells had best ITDs $<135 \mu$ s. Moreover, best ITDs depended significantly on BF in that neurons with lower BFs exhibited longer best ITDs (cc, -0.66 ; $p<0.001$ ) (supplemental Fig. $2 A$, available at www.jneurosci.org as supplemental material). In contrast, sensitivity to modulations in ITD was maximal ("ITD of steepest slope," first derivative of the ITD function) within the physiological range of ITDs for the majority of ITD functions ( 39 of 56) and was independent of BF (correlation coefficient, $-0.16 ; p>0.293$ ) (supplemental Fig. 2B, C, available at www.jneurosci.org as supplemental material).

Measurements of ITD tuning are typically performed at only one intensity (20 or $30 \mathrm{~dB}$ above threshold). However, little is known about the influence of absolute intensity on ITD tuning of MSO neurons. Therefore, we also obtained ITD functions over a wide range $(35 \mathrm{~dB})$ of absolute sound intensities (ASIs) and assessed best IPDs at each of the intensities. We successfully tested this paradigm on $36 \mathrm{MSO}$ neurons, and an example recording of one MSO neuron is provided in Figure 2A1: Clearly, the maximum number of spikes increased with increasing ASI. In contrast, the best IPD of the neuron was unaffected by changing the ASI. When plotting the best IPDs against the corresponding ASIs, the slope of a linear regression fit was only -0.001 phase $/ \mathrm{dB}$, indicating robustness of best IPD against variations in ASI (Fig. 2A2). As summarized in Figure 2 B1, the best IPD of the majority of neurons did not change at all or just slightly with increasing intensity. This high robustness was also exhibited when expressed in time delays, because shifts of best ITD were typically less than \pm 10 $\mu \mathrm{s} / \mathrm{dB}$ (Fig. 2 B2).

\section{Pharmacological experiments}

MSO neurons receive prominent inhibitory, glycinergic inputs, mainly from the MNTB and, to a lesser extent, from the LNTB (Cant and Hyson, 1992; Kuwabara and Zook, 1992; Grothe and Sanes, 1993; Kapfer et al., 2002). To test the influence of the glycinergic inhibition on ITD sensitivity, we iontophoretically applied either the glycine antagonist strychnine or glycine itself onto MSO neurons while presenting different ITDs at the BFs of the cells. These are very difficult experiments, potentially because of the action potential-producing zone being rather distant up the axon (Scott et al., 2007). Nevertheless, we successfully applied drugs in 17 MSO recordings in which we assessed ITD functions before and during drug application. We were able to record from 13 cells long enough to achieve full recovery of the original ITD sensitivity after stopping drug application (supplemental Table 1, available at www.jneurosci.org as supplemental material). In 6 of the 17 MSO neurons, we tested the effect of blocking glycinergic inhibition via strychnine on ITD functions (Fig. 3). We first focus on the total response, including onset and ongoing components. Control functions before application are shown in solid blue, whereas solid red lines indicate the response functions for the
A2

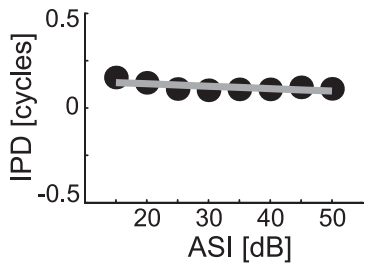

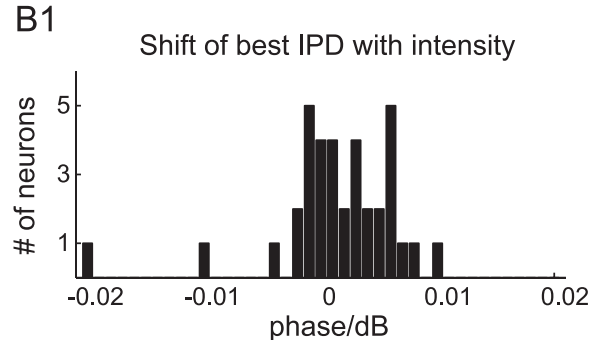

B2

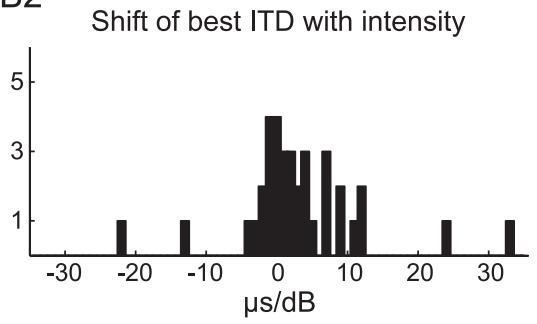

Figure 2. ITD sensitivity is robust against variations in ASI. A1, Responses of an example neuron that exhibited ITD sensitivity over the full test range of ASIs. Frequency of pure-tone stimulation was $683 \mathrm{~Hz}$ (BF). ASIs in decibels SPL are given in the top right ASIs. The gray line depicts a linear fit as a basis for the histograms showing shifts in IPD (B1) and ITD (B2) for 36 neurons. Values are clustered around 0 in both measures, indicating high robustness of best IPD/ITD against changes in ASI.

strychnine condition. Note that four neurons were tested with pure tones at $\mathrm{BF}$, whereas two neurons responded best to narrowband pulses centered on BF (cell 061500mso07, bandwidth, $\pm 500 \mathrm{~Hz}$; cell $100101 \mathrm{mso} 03$, bandwidth, $\pm 300 \mathrm{~Hz}$; BFs are given in panels). In addition to the expected increase in overall spike rate, at least at some ITDs, the slopes that were closest to 0 ITD shifted to the left (toward zero or more negative ITDs) in all six cells tested. Data of one additional cell in which strychnine also induced a similar shift are not shown, because its location could not be verified. Response types were not changed by blocking inhibition. Circular statistics for analyzing the central period of the ITD functions ( \pm 0.5 IPD centered on best IPD) revealed that the change in response distribution within the central peak of the ITD function was significant in five of the six cells $(p<0.05$, Watson's $U^{2}$ test) (total response: Fig. 3, filled asterisks). That the obvious shift in cell $100101 \mathrm{mso} 04$ was not significant $(0.2>p>$ 0.1 , Watson's $U^{2}$ test) is partially attributable to the fact that spike counts in this cell were low (and slightly but steadily decreased during the recordings) and its onset component was not ITD sensitive (in contrast to its ongoing component, which shifted significantly) (see below). Values of best IPD before and during strychnine application are compiled in supplemental Table 1 (available at www.jneurosci.org as supplemental material). Note that best ITDs are not necessarily equivalent to the apparent peaks of the ITD functions, but are derived from calculation of the mean vector (see Materials and Methods) that reflects the overall shape of the central period of the ITD functions. Normalizing the shifts in best ITD for the BF of the cells, the average difference in best IPD between control and strychnine condition was 0.13 (SD, \pm 0.08 ) cycles (scatter plot in Fig. 3, filled circles). Recoveries were similar to best IPDs under control conditions with an average difference of 0.02 cycles $(S D, \pm 0.06)$. For explanation of the apparent lack of a shift in the "side peak" of the 

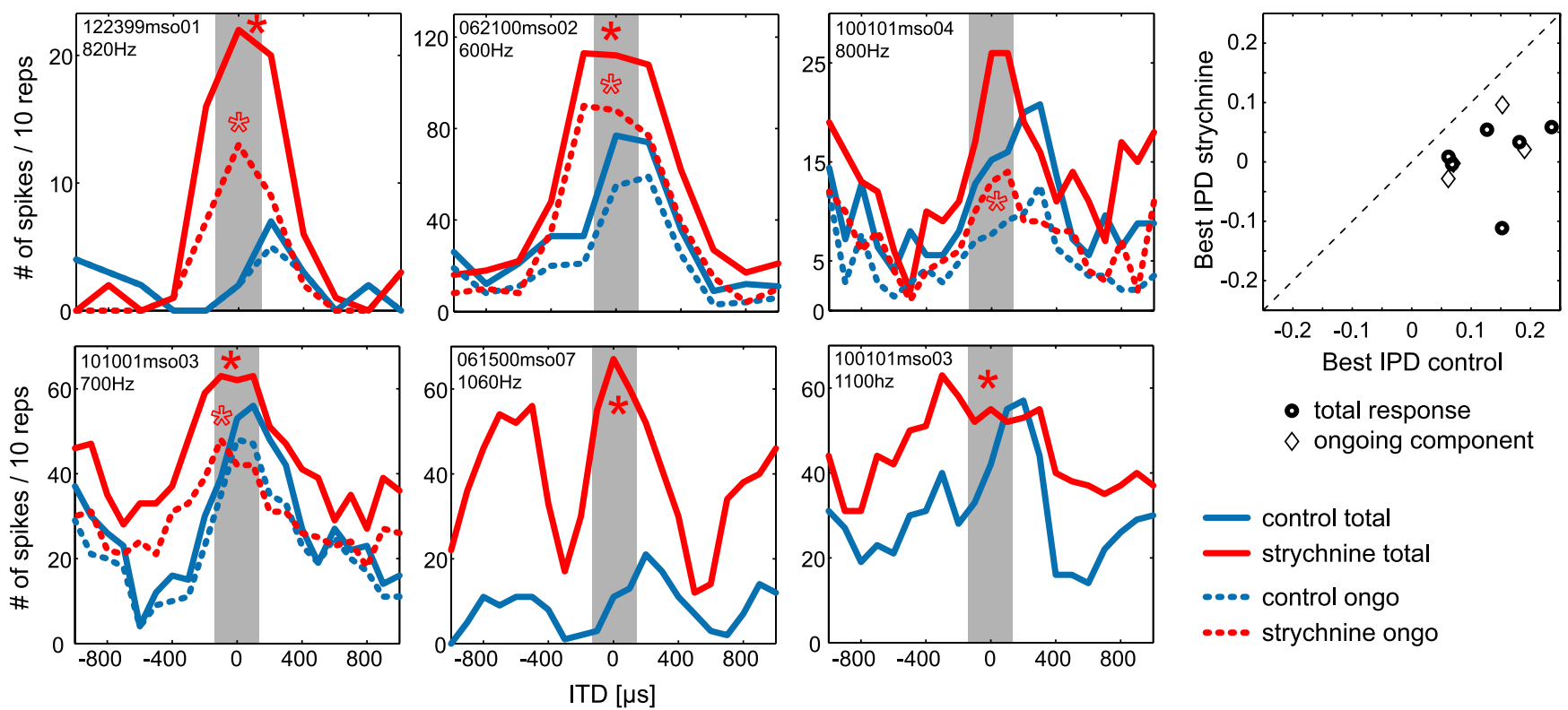

- total response

$\diamond$ ongoing component

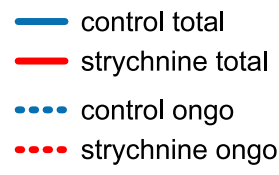

Figure 3. Strychnine induces shifts in response distribution toward 0 ITD for total and ongoing response. ITD functions for pure-tone stimulation at BF (provided at top left of panels) for four neurons and for trains of narrowband pulses centered on BF for two neurons (middle and right of bottom panels) are shown before and during or shortly after local strychnine application. The solid blue lines indicate control condition of the total responses of the cells, whereas the corresponding strychnine conditions are depicted by the solid red lines. Ongoing components of the responses are shown in the dashed lines. The asterisks mark significant shifts for total (filled asterisks) or ongoing response (open asterisks). The gray bar indicates "physiological range" of ITDs ( $\pm 135 \mu \mathrm{s}$ ). The scatter plots in the rightmost panel show changes in best ITD during strychnine application for each cell.

response of neuron $061500 \mathrm{mso} 07$, see supplemental Figure 3 (available at www.jneurosci.org as supplemental material).

Responses were typically strongest at the beginning of stimulation and weaker during the ongoing component. Potentially, the effects of strychnine application could be different for the ongoing component of the response or even restricted to the stronger onset response. Therefore, we tested whether blocking glycinergic inhibition equally affects ITD sensitivity of all response components. Four of the six cells showed a clear ongoing component in their response to ITDs, which we analyzed separately by removing the first $10 \mathrm{~ms}$ of the response. The same overall effect of strychnine, namely a shift of the response distribution to the left, was apparent for these ongoing responses. In Figure 3, the ITD functions of the ongoing components are shown in dashed lines (blue lines depict control; red lines depict strychnine conditions). Importantly, the shifts in response distribution toward 0 ITD were significant for all four neurons $(p<$ 0.05 , Watson's $U^{2}$ test). Figure 4 exemplifies the effect by showing one neuron in which ITD sensitivity for ongoing responses (Fig. $4 A$, blue dots in the dot raster plots) was identical with the ITD sensitivity of the total response (compare Fig. 3, bottom left panel). Figure $4 B$ shows the ITD functions of the ongoing response, which clearly shifted because of blocking of inhibition (red line) by firing at higher rates at ipsilateral leading ITDs, which were "unfavorable" before strychnine application as well after recovery (dashed gray line). In Figure $4 C$, significant mean phase angles of the phase-locked response to the pure-tone frequency of the ongoing responses before and during strychnine application are presented as dashed lines. Note that, in particular when the contralateral stimulus was leading (positive ITD values), the mean angle decreased; hence the neuron tended to respond earlier within each period of the pure tone than under control (or recovery) (data not shown) conditions. For three ITDs tested, this effect was statistically significant $(p<0.05$, Watson's $U^{2}$ test). The vector strength of the ongoing compo- nent was very high with and without strychnine application (Fig. $4 C$, solid lines). The same effect was found in a second neuron (062100mso02) with well phase-locked, ongoing responses yielding a sufficient number of spikes to perform this analysis.

The findings from strychnine application demonstrated that glycinergic inhibition is strongly involved in internally delaying the coincidence of the net excitation of the binaural inputs, thereby tuning the ITD functions of MSO neurons in relation to the physiological range. The shifts of best ITD in the ongoing components further showed that the effect of inhibition is not restricted to the onset, but that it is active throughout the duration of sound stimulation. These experiments, however, cannot distinguish between two principle possibilities that explain how this tuning is achieved by glycinergic inhibition: (1) Stimulus driven but tonic inhibition (accumulating over cycles) could change the sensitivity of the MSO neurons to coincidence of excitatory inputs (cf. Zhou et al., 2005). (2) The MNTB projection could provide well timed, contralaterally driven phase-locked inhibition that interacts with the timing of the contralaterally driven excitation on a cycle-by-cycle basis (cf. Grothe, 2003). The basic principle of this hypothesis is an earlier arrival of the IPSP relative to the EPSP, which effectively delays the net PSP from the contralateral side. Thereby the binaural coincidence window is not only shortened but also shifted to more positive ITDs (contralateral stimulus leading) (Fig. 7A). These two hypotheses make distinct predictions, which can be experimentally tested: (1) If timing of inhibition does not matter, tonically applied glycine would either enhance the effect of the tonic synaptic inhibition and shift the ITD function even further to the right (Zhou et al., 2005) or, alternatively, would simply lower the response rate because of a hyperpolarization of the cell. (2) If timing of inhibition does matter, tonic application of glycine would diminish the effectiveness of endogenous inhibition by "masking" the discrete events of the phase-locked synaptic inhibition (Fig. 7C). As a result, ITD functions would also shift to the "left" (more negative 
ITDs) but the sides of the ITD functions would be affected differently from those during strychnine applications. Blocking inhibition with strychnine would broaden the coincidence window by expanding it to more ipsilateral leading ITDs, which in turn would increase neuronal response rates mainly at ITDs to the left of the original best ITD (Fig. 7B). This prediction is consistent with our findings shown above. In contrast, tonic glycine application induces constant hyperpolarization in the neurons, which in turn would not only mask the timing of the endogenous inhibition, but, at the same time, only a smaller fraction of the net PSP would exceed threshold for binaural coincidence, effectively cutting off the declining tail of the contralateral net PSP. Hence, the accompanied shift in ITD tuning would be predominately created by decreased response rates at the right-hand side of the ITD function (Fig. 7C).

To test these hypotheses, we used iontophoresis to tonically apply glycine onto MSO neurons while presenting pure tones at $\mathrm{BF}$ with varying ITDs. Because the neurons were very sensitive to glycine application, we carefully controlled the amount of glycine so that the neurons were not silenced but their spike rate was lowered. This was successfully achieved in 11 of 12 peak-type neurons tested. In one neuron (data not shown), a slight reduction of spike counts was correlated with only a marginal shift in best ITD. Here, apparently, drug application was not strong enough to yield any quantifiable effects. In 9 of the 11 cells with clear drug-induced effects shown in Figure 5, tonic glycine application caused a significant shift of the ITD function toward the left $(p<0.05$, Watson's $U^{2}$ test). Expressed in phase differences to normalize for frequency, overall the best IPDs during glycine application were significantly smaller than the control best IPDs ( $p<0.01$, one-sided paired Student's $t$ test). The mean difference between control best IPD and the best IPD for glycine condition for 11 cells was $0.10 \pm 0.10$ (SD) (Fig. 5, scatter plot; supplemental Table 1, available at www.jneurosci.org as supplemental material). In contrast, the average difference between control $(n=11)$ and recovery best IPD $(n=8)$ was only 0.02 (SD, \pm 0.03 ). Hence tonic application of glycine caused ITD functions to shift to smaller or more negative best ITDs. Three neurons were additionally tested with a $\mathrm{NaCl}$ solution $(1 \mathrm{M})$ with $\mathrm{pH} 3$, similar to the $\mathrm{pH}$ of the drug solutions, to control for possible effects of acidity changes in the extracellular medium on IPD functions. In all cells tested, $\mathrm{NaCl}$ application alone did not cause a shift of IPD functions (average difference in best IPD, 0.003) (Fig. 5, black lines) (Watson's $U^{2}$ test, $p>0.2$ ), confirming that the drug-induced changes in IPD tuning were specific.

Glycine strongly reduced the ongoing components of the responses. As a consequence, most neurons only responded during the initial $10 \mathrm{~ms}$ of a stimulus. Therefore, we analyzed the spikes after the initial $2 \mathrm{~ms}$ of the response as ongoing component, which, following our results shown above (Fig. $1 F$ ), seems to be an appropriate alternative approach. This way we could analyze significant ongoing responses during glycine application in four cells. Analogous to strychnine, the shifting effect of glycine was also apparent for the ongoing responses alone (Fig. 5, top row, dashed lines) ( $p<0.05$, Watson's $U^{2}$ test; $n=3$ ). Ongoing responses remained phase-locked during glycine application, displaying an average VS of 0.58. A detailed example for the effect of glycine is shown in Figure 6. The raster plots show highly synchronized discharges to pure-tone stimulation $(630 \mathrm{~Hz})$ for both onset and ongoing responses for control conditions and during application of glycine.

We showed above that both strychnine and glycine application induced left shifts in the overall ITD sensitivity. These findings do not confirm the hypothesis of endogenous glycinergic inhibition acting tonically (Zhou et al., 2005). Instead, the data are in line with the predictions made by the timed inhibition hypothesis (Fig. 7). Specifically, the timed inhibition hypothesis holds that, under normal conditions, ipsilateral stimulation 

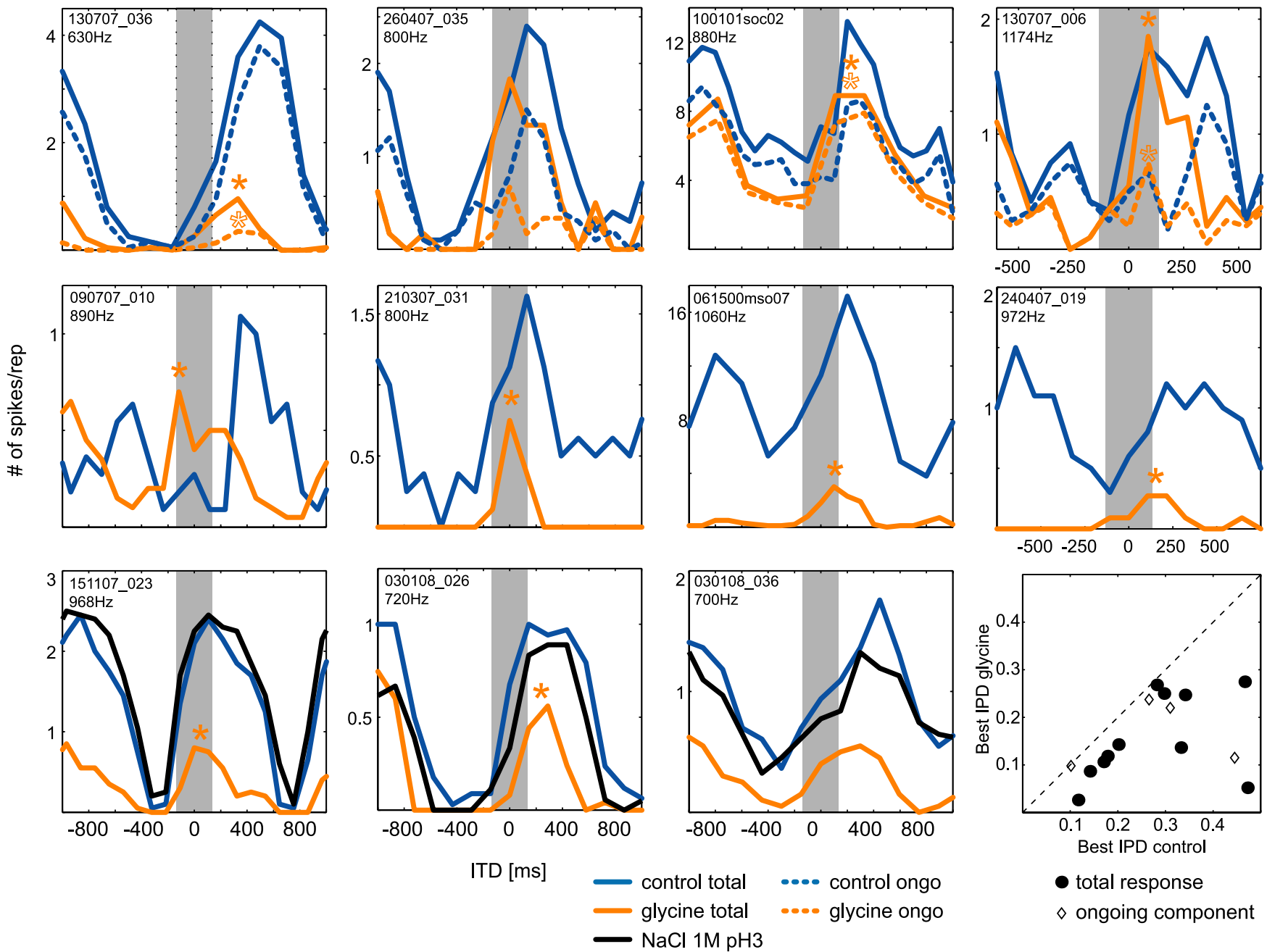

- total response

$\diamond$ ongoing component

Figure 5. Tonic inhibition shifts the best ITD toward 0 ITD. ITD functions for pure-tone stimulation at BF (provided at top left of panels) for 11 neurons are shown before (solid blue lines) and during tonic glycine application (solid orange lines). During glycine application, response distributions were significantly shifted in 9 of 11 cells (filled asterisks). Shifts of best ITDs were also present in the ongoing responses (dashed blue and orange lines for control and during application, respectively). The solid black lines in the bottom panels show the ITD function during application of $\mathrm{NaCl}$ solution with similar acidity as the glycine solution, $\mathrm{pH}$ 3. "Physiological range" of ITDs is marked by gray bars. The scatter plot in the bottom-most right panel summarizes the shifts in best IPD for all 11 neurons.

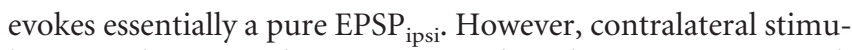
lation evokes not only an EPSP contra $_{\text {but also an IPSP }}$ contra with shorter onset latency preceding the EPSP $\mathrm{P}_{\text {contra. }}$. The summation of the IPSP contra $_{\text {and }}$ EPSP $_{\text {contra }}$ generates a contralaterally evoked PSP with two components, an early hyperpolarization followed by a depolarization (Fig. 7A1). Therefore, the net excitation is delayed and binaural coincidence of the $\mathrm{EPSP}_{\mathrm{ipsi}}$ and contralaterally evoked net excitation only occurs for stimulations with the contralateral sound leading (Fig. 7A2). Consequently, the peak of the IPD function is at positive values (Fig. 7A3).

The changes in IPD functions caused by blocking inhibition with strychnine or tonically enhancing inhibition with glycine can be explained by the effects of the drugs on the delays imposed on the net excitation by contralaterally evoked inhibition. The effects of blocking inhibition with strychnine are straightforward because strychnine simply eliminates the IPSP contra $_{\text {; }}$ hence the net excitation (Fig. 7B1, PSP $_{\text {contra }}$ ) is now advanced in time and broadened compared with the net excitation in the control condition (Fig. 7B1, arrow). Therefore the IPD functions are shaped only by the coincidence of the contralateral and ipsilaterally evoked EPSPs (Fig. 7B2). Thus, the IPD function is shifted to the left, mainly by expanding on the left-hand side (ipsi ear leading)
(Fig. 7B3, arrow), just like the contralateral EPSP expanded on the left-hand side compared with the control net excitation (Fig. $7 A 2)$.

The tonically applied glycine, however, greatly reduces or even eliminates the efficacy of the IPSP ${ }_{\text {contra }}$ because the membrane potential is already at a hyperpolarized value (Fig. 7C1, black arrow and orange dotted line). Thus, the $\mathrm{PSP}_{\text {contra }}$ is reduced in overall amplitude but also advanced compared with the control condition. Coincidence of the PSP $\mathrm{P}_{\text {contra }}$ and the ipsi EPSP is now also occurring at IPDs near 0 cycles, analogous to strychnine application (Fig. 7C2). However, the induced reduction of the EPSP amplitude has a pronounced impact on the right-hand side of the IPD function (Fig. 7C1, arrow), because the more negative PSP is insufficient for reaching binaural threshold at large contralateral IPDs. Hence the combination of these two effects (the

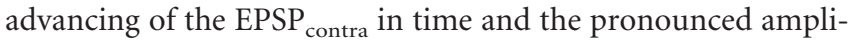
tude reduction) results in a left-shifted IPD function that is narrowed on the right-hand side and has overall reduced spike rates compared with control (Fig. 7C3). Note that constant hyperpolarization by glycine alters the membrane conductance of the cells. This could lead to additional accelerations of the PSP kinet- 
ics that would even add to the narrowing effect of tonic glycine application.

Together, the timed inhibition hypothesis predicts that during strychnine application IPD function should broaden at the left-hand side, whereas tonic glycine application should mainly induce a narrowing of the right-hand side of the IPD function. We normalized the IPD functions of all cells presented in Figures 3 and 5 to their respective control and averaged them within conditions (for details, see figure legends). Intriguingly, the mean IPD functions for control, strychnine, and glycine conditions closely resemble the qualitative predictions of the timed inhibition scenario (Fig. 7D1,D2): During strychnine application, the mean IPD function broadened mostly to the left-hand side, whereas glycine application restricted the mean IPD function mainly on the righthand side of the control function. This qualitative result could also be statistically verified by normalizing all IPD functions individually (thereby ignoring the druginduced overall change in spike rate) and testing for significant differences at each IPD presented. For the population of cells tested with strychnine, normalized response rates were significantly higher during drug application compared with control conditions for IPDs composing the left-hand side ( $p<0.001$, one-sided Student's $t$ test), but not for IPDs on the right side of the original best IPD ( $p>0.5$, twosided Student's $t$ test) (for details, see Materials and Methods). Accordingly, compared with control conditions, normalized response rates were significantly lower during glycine application for IPDs composing the right-hand side ( $p<0.0001$, one-sided Student's $t$ test), but did not change for IPDs on the left side ( $p>0.3$, two-sided Student's $t$ test) (for details, see Materials and Methods). Together, these results strongly support the hypothesis of endogenous glycinergic inputs being well timed, discrete events as illustrated in Figure $7 A$ and are inconsistent with a nonspecific, tonic effect of inhibition.

\section{Discussion}

There are three main findings in the present study: (1) The ITD tuning of gerbil MSO neurons is coherent with the idea that ITDs are encoded via a population rate code: Best ITDs depended on the neuronal BFs and were mostly outside the "physiological range" of ITDs, whereas most of the steepest slopes of the ITD functions fall within this range. (2) Blocking glycinergic inhibition with strychnine enhanced the spike rates and retuned ITD sensitivity by significantly shifting response distributions of MSO neurons toward 0 ITD and slopes out of the "physiological range." (3) Tonic application of glycine reduced the spike rates but at the same time also induced shifts of the ITD functions toward 0 ITD, albeit with characteristics different than those observed by strychnine application.

\section{Basic ITD properties}

MSO neurons responded preferentially to contralateral leading ITDs, corroborating previous findings from both the MSO (Goldberg and Brown, 1969; Crow et al., 1978; Yin and Chan, 1990; Spitzer and Semple, 1995) and higher, subcortical structures (McAlpine et al., 2001; Hancock and Delgutte, 2004; Siveke et al., 2006, 2007). Such positions of the peaks of the ITD functions result in their slopes being positioned within the physiological range of ITDs (McAlpine et al., 2001; Harper and McAlpine, 2004). Importantly, these data corroborate the hypothesis that the population of MSO neurons encode various locations along the azimuthal plane by a monotonic modulation of their output rate along the slopes of their ITD function (McAlpine and Grothe, 2003; Palmer, 2004).

The fraction of neurons we found to respond only to the onset of stimuli was high, which could partially be attributable to anesthesia. Kuwada et al. (1989) showed prominent effects of anesthesia on neuronal response characteristics in the rabbit midbrain. However, a recent study in the gerbil found that anesthesia-induced changes of temporal response characteristics were restricted to higher auditory centers (Ter-Mikaelian et al., 2007). Possibly the high percentage of onset responses reflects a behavioral relevance, because many natural signals have prominent onset components. To date, the relative contributions of onset and ongoing components for low-frequency sound localization is a matter of debate (Buell et al., 1991; Akeroyd and 


\section{A control condition: with timed inhibition}

A1

A2

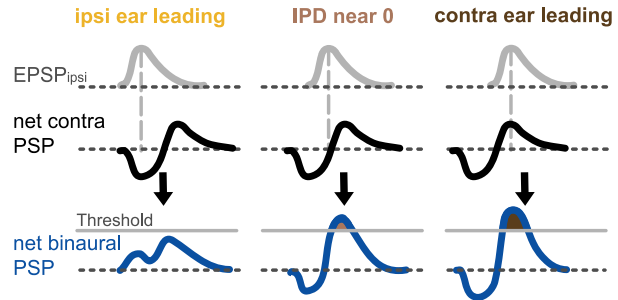

B endogenous inhibition blocked with strychnine

B1

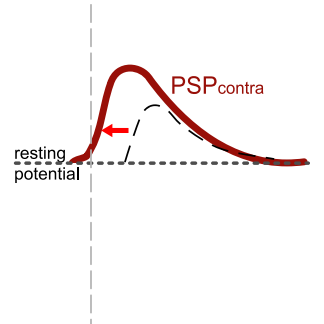

B2

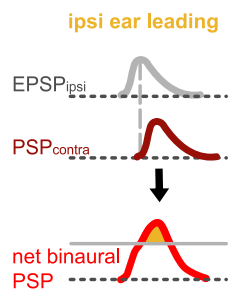

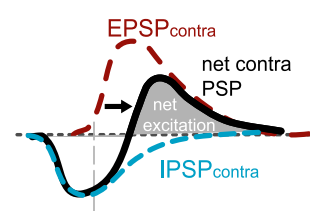

A3

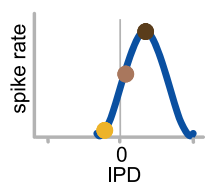

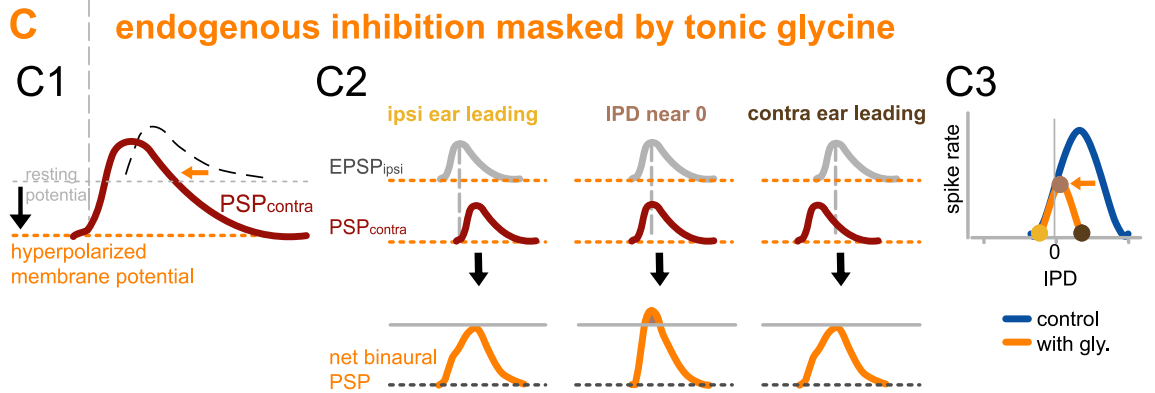

D1 scheme summary

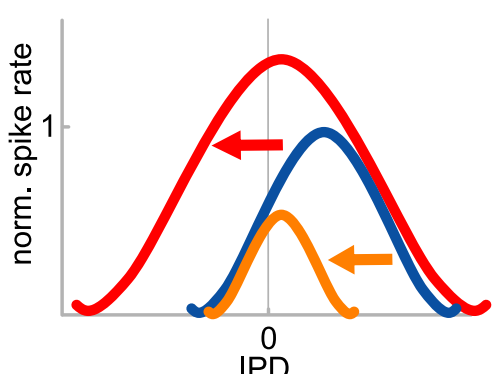

IPD
D2

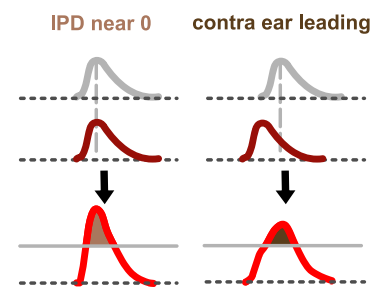

B3

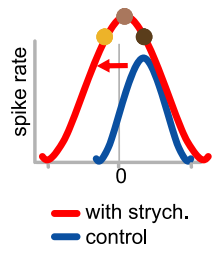

Bernstein, 2001; Best et al., 2004). Importantly, at the level of the MSO, both onset and ongoing components exhibit similar ITD sensitivity and this ITD sensitivity is equally dependent on glycinergic inhibition (see below).

Obtaining ITD functions at multiple intensities changed overall response rates but resulted in either no or only small shifts in best ITDs. These findings are in line with previous reports from the dog MSO as well as the cat midbrain (Goldberg and Brown, 1969; Yin and Kuwada, 1983). Interestingly, in their seminal work in 1969, Goldberg and Brown argued that the invariance of best ITD with intensity is closely linked to inhibitory influences. It therefore seems conceivable that the hyperpolarizing inhibitory inputs in the MSO help to convey intensity robustness of ITD sensitivity by defining the binaural coincidence window and preventing outof-phase responses, which otherwise increase in likelihood at higher intensities (Reed and Durbeck, 1995). Such gain control is also present in the avian analog of the MSO; however, it is achieved by tonic, GABAergic inputs providing depolarizing, shunting inhibition (Yang et al., 1999; Burger et al., 2005; Dasika et al., 2005).

\section{Pharmacology}

Because it is inherently difficult to record from MSO cells (Guinan et al., 1972), our sample size with pharmacology is small. However, in all cells in which firing rates were increased by antagonizing glycinergic inhibition with strychnine, the left side of the ITD functions shifted markedly toward 0 ITD. Similarly, whenever glycine application reduced firing rates, correlated shifts in the right-hand side of the ITD functions were observed. Importantly, in all neurons with an ongoing response, ITD functions were shifted by application of glycine or strychnine. Thus, onset and ongoing components seem to be tuned via the same circuitry and synaptic properties. It follows that glycinergic inhibition is an Contralateral PSP (black line). The basic principle of the timed inhibition hypothesis is that the contralateral IPSP ${ }_{\text {contra }}$ (light blue dashed line) precedes the contralateral EPSP (brown dashed line) on a cycle-by-cycle basis. This causes a delay of the contralaterally induced net excitation (shaded area under black line). A2, Binaural interaction (blue) of the net contra PSP (black) with the EPSP $_{\text {ipsi }}$ (gray) at different IPDs. Maximal coincidence of the net excitations from both ears occurs for contra leading IPDs (rightmost column of panels) compensating for the inhibition-induced delay of the contralateral excitation. $\boldsymbol{A}$, Resulting IPD function: yellow-, light brown-, and dark brown-filled circles correspond to the three stimulus conditions shown in $\boldsymbol{A 2}$. B, Effect of strychnine application. B1, Experimentally blocking the timed inhibition with strychnine results in a net contra PSP that is identical with the contralateral EPSP (brown line; compare with brown dashed line in A1). Hence the net contra PSP is advanced in time and broadened (indicated by red arrow) compared with the control condition (dashed black line). B2, Maximal binaural coincidence during strychnine application occurs at IPDs near 0 cycles (middle column of panels). Ipsilateral leading IPDs also create suprathreshold net binaural PSPs (left column of panels) because of partial overlap of ipsi and contra excitation. This results in overall increased response rates and a broadening of the IPD function on the left-hand side, shifting the peak toward 0 IPD (B3). C, Effect of tonic glycine application. C1, Tonic glycine application induces a tonic hyperpolarization (black arrow and orange dotted line), which has two important effects. First, the timed inhibition is masked; hence the net PSP ${ }_{\text {contra }}$ is not delayed but, second, reduced compared with the strychnine condition. Compared with control condition, this reduction has the most pronounced impact at the

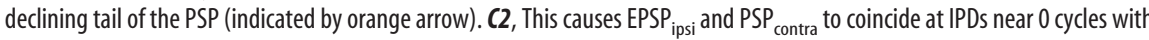
only a small fraction of the net binaural PSPs exceeding threshold. C3, Compared with control conditions, the right-hand side of

$\leftarrow$

the IPD function is highly reduced causing a left shift of the overall IPD function (orange arrow). D1, D2, These qualitative predictions are summarized in $\mathbf{D} \mathbf{1}$ and are consistent with the results in our population data as depicted in $\mathbf{D 2}$, showing the average normalized spike rates of control $(n=15)$, strychnine ( $n=6)$, and glycine $(n=11)$ condition (shown by asterisks, diamonds, and filled circles, respectively). For averaging, responses were binned in IPD widths of 0.125 cycles. Average normalized IPD functions were derived from these mean values by Gaussian fits. The $R^{2}$ values of the fits were $0.92,0.98$, and 0.92 for the control (blue), strychnine (red), and glycine (orange) conditions, respectively. 
important mechanism for tuning ITD functions in the mammalian MSO.

There are at least two ways in which inhibitory inputs could interfere with coincidence detection of binaural excitation, both causing the contralateral inputs to be functionally delayed. One possibility was proposed by Zhou et al. (2005) and assumes both tonic inhibition and dendritic asymmetry [i.e., that the axons of MSO cells originate from the lateral dendrites (which receive the ipsilateral excitatory inputs) (Smith et al., 1993)]. The basic idea is that tonic inhibition delays the propagation of EPSPs from the contralateral input to the spike-initiating zone but does not affect ipsilateral EPSPs. However, most MSO cells are not asymmetric (Scott et al., 2005) (our unpublished observations). Additionally, our experiments showed that glycine application did not enhance the effect of endogenous synaptic inhibition, as the model would suggest, but reduced it.

A second hypothesis, proposed by Brand et al. (2002), is that precise timing of the inhibitory inputs relative to the excitatory inputs shifts the coincidence window as explained in Figure 7. The hypothesis predicts not only the shifting of ITD functions to more ipsilateral ITDs for both strychnine and tonic glycine application but also that different sides of the ITD functions are changed by glycine compared with strychnine. We emphasize that the glycine-induced shifts are not attributable to receptor desensitization, because spike rates were lowered throughout glycine application; hence the cells were hyperpolarized. Theoretically, because of the additional opening of chloride channels, this hyperpolarization might have accelerated the kinetics of the sound-evoked PSPs. However, synaptically evoked EPSPs measured in the gerbil MSO in vitro are essentially as fast as the underlying EPSCs (Magnusson et al., 2005; Scott et al., 2005) leaving not much space for additional acceleration. Also, we emphasize that the acceleration of PSP kinetics by tonic hyperpolarization would affect ipsilateral and contralateral inputs equally, hence would cause a narrowing of the ITD function but cannot account for the observed shifts. Turecek and Trussel (2001) described presynaptic glycine receptors that enhance neurotransmission in the calyx of Held. In principle, presynaptic glycine receptors could also be present in the MSO, which might be affected by the glycine application. However, glycinergic inputs to the MSO are restricted to the somatic area and are absent in the dendrites (Kapfer et al., 2002). Hence, presynaptic, glycine-mediated effects would affect the inhibitory inputs and would counteract the postsynaptic effects of glycine iontophoresis. Therefore, we regard this as an unlikely explanation.

Thus, our data strongly favor the scenario involving well timed inhibition. This scenario includes two critical features: One feature is the specificity in the relative onset of inhibition and excitation, whereas the other is the time course of the inhibition. With regard to timing, the scenario incorporates contralateral inhibitory inputs that arrive a few hundred microseconds before the contralateral excitatory inputs. Our data support this feature because the inhibitioninduced delay in the mean phase angle of the response that was exposed by strychnine application (Fig. 4C) was exactly in this range. Moreover, the MNTB and its MSO inputs exhibit extreme specializations for exact timing and speed of transduction (Kapfer et al., 2002; von Gersdorff and Borst, 2002). For instance, it is well established that the MNTB-mediated inhibition via the even longer pathway to the lateral superior olive coincides or even precedes the excitation via the much shorter ipsilateral pathway (Grothe and Park, 1995; Tollin and Yin, 2005). Most importantly, in vivo recordings in the bat MSO revealed contralateral inhibition to have the capacity to arrive simultaneously or even precede the contralateral excitation (Grothe, 1994; Grothe and Park, 1998). Finally, artificial, simulta- neous stimulation of both the excitatory and inhibitory inputs from the contralateral side in vitro resulted in full spike suppression in $>80 \%$ of gerbil MSO neurons (Grothe and Sanes, 1993). Hence the MNTB pathway is able to compensate for the delay because of the additional synapse. The second feature is the exact time course of the inhibitory inputs, which in the model acted on a cycle-by-cycle basis. Although we could not measure the time course of inhibition directly, our findings indicate such a phasic interaction of inhibitory and excitatory inputs. Notably, our sample for pharmacological manipulations is restricted to neurons with CFs $<1200 \mathrm{~Hz}$, which represents the dominant frequency range of MSO cells (Yin and Chan, 1990; Spitzer and Semple, 1995). We cannot exclude the possibility that the effect of inhibition as described above is restricted to this frequency range and that inhibition is not fast enough to tune ITD sensitivity at higher frequencies. Interestingly, in our total sample of MSO recordings, we found several ITD-sensitive cells with higher CFs (up to $4800 \mathrm{~Hz}$ ), but their BFs were all $\leq 1200 \mathrm{~Hz}$. In vitro studies on isolated glycinergic currents and potentials determined kinetics that, although very fast, were on average not fast enough to follow each stimulation cycle at higher frequencies (Smith et al., 2000; Magnusson et al., 2005). However, the effective period of glycinergic inhibition determined in MSO brain slice experiments using multiple inputs was $<1 \mathrm{~ms}$ (Grothe and Sanes, 1994). Recently, it was shown in MNTB membrane patches that the kinetics of glycinergic inhibition is accelerated by the corelease of GABA (Lu et al., 2008). Hence, it is currently unclear how fast inhibition can act in vivo and how fast it would have to be to make our scenario feasible [note that Brand et al. (2002) only aimed for proof of principle, not for assessing the kinetics required].

The present data provide strong evidence for glycinergic inhibition as a key factor in setting functional delays of the binaural excitatory inputs, but it is important to note that glycine might act in concert with other factors that could cause internal delays of the MSO inputs (cf. Joris and Yin, 2007). These factors include axonal length (Jeffress, 1948; Smith et al., 1993; Beckius et al., 1999), cochlear delays if frequency tuning of the different inputs do not exactly match (Shamma et al., 1989; Joris et al., 2006), or differences in myelination or axonal diameter. Additional research is needed to address these questions using more sophisticated manipulations such as experimentally induced decorrelation of the phase-locked inhibitory inputs.

\section{References}

Adams JC (1977) Technical considerations on use of horseradishperoxidase as a neuronal marker. Neuroscience 2:141-145.

Akeroyd MA, Bernstein LR (2001) The variation across time of sensitivity to interaural disparities: behavioral measurements and quantitative analyses. J Acoust Soc Am 110:2516-2526.

Batra R, Kuwada S, Fitzpatrick DC (1997) Sensitivity to interaural temporal disparities of low- and high- frequency neurons in the superior olivary complex. I. Heterogeneity of responses. J Neurophysiol 78:1222-1236.

Beckius GE, Batra R, Oliver DL (1999) Axons from anteroventral cochlear nucleus that terminate in medial superior olive of cat: observations related to delay lines. J Neurosci 19:3146-3161.

Best V, van Schaik A, Carlile S (2004) Separation of concurrent broadband sound sources by human listeners. J Acoust Soc Am 115:324-336.

Brand A, Behrend O, Marquardt T, McAlpine D, Grothe B (2002) Precise inhibition is essential for microsecond interaural time difference coding. Nature 417:543-547.

Buell TN, Trahiotis C, Bernstein LR (1991) Lateralization of low-frequency tones: relative potency of gating and ongoing interaural delays. J Acoust Soc Am 90:3077-3085.

Burger RM, Cramer KS, Pfeiffer JD, Rubel EW (2005) Avian superior olivary nucleus provides divergent inhibitory input to parallel auditory pathways. J Comp Neurol 481:6-18.

Cant NB (1991) Projections to the lateral and medial superior olivary nuclei 
from the spherical and globular bushy cells of the anteroventral cochlear nucleus. In: Neurobiology of hearing: the central auditory system (Altschuler RA, Bobbin RP, Clopton BM, Hoffman DW, eds), pp 99-119. New York: Raven.

Cant NB, Hyson RL (1992) Projections from the lateral nucleus of the trapezoid body to the medial superior olivary nucleus in the gerbil. Hear Res 58:26-34.

Carr CE, Soares D (2002) Evolutionary convergence and shared computational principles in the auditory system. Brain Behav Evol 59:294-311.

Clark GM (1969) Vesicle shape versus type of synapse in the nerve endings of the cat medial superior olive. Brain Res 15:548-551.

Crow G, Rupert AL, Moushegian G (1978) Phase locking in monaural and binaural medullary neurons: implications for binaural phenomena. J Acoust Soc Am 64:493-501.

Dasika VK, White JA, Carney LH, Colburn HS (2005) Effects of inhibitory feedback in a network model of avian brain stem. J Neurophysiol 94:400-414.

Goldberg JM, Brown PB (1969) Response of binaural neurons of dog superior olivary complex to dichotic tonal stimuli: some physiological mechanisms of sound localization. J Neurophysiol 32:613-636.

Grothe B (1994) Interaction of excitation and inhibition in processing of pure tone and amplitude-modulated stimuli in the medial superior olive of the mustached bat. J Neurophysiol 71:706-721.

Grothe B (2003) New roles for synaptic inhibition in sound localization. Nat Rev Neurosci 4:540-550.

Grothe B, Park TJ (1995) Time can be traded for intensity in the lower auditory system. Naturwissenschaften 82:521-523.

Grothe B, Park TJ (1998) Sensitivity to interaural time differences in the medial superior olive of a small mammal, the Mexican free-tailed bat. J Neurosci 18:6608-6622.

Grothe B, Sanes DH (1993) Bilateral inhibition by glycinergic afferents in the medial superior olive. J Neurophysiol 69:1192-1196.

Grothe B, Sanes DH (1994) Synaptic inhibition influences the temporal coding properties of medial superior olivary neurons: an in vitro study. J Neurosci 14:1701-1709.

Grothe B, Fritzsch B, Köppl C, Casseday JH, Carr CE (2004) The evolution of central pathways and their neural processing patterns. In: Evolution of the vertebrate auditory system (Manley GA, Popper AN, Fay RR, eds), pp 289-359. New York: Springer.

Guinan JJ Jr, Norris BE, Guinan SS (1972) Single auditory units in the superior olivary complex. II. Locations of unit categories and tonotopic organization. Int J Neurosci 4:147-166.

Hancock KE, Delgutte B (2004) A physiologically based model of interaural time difference discrimination. J Neurosci 24:7110-7117.

Harper NS, McAlpine D (2004) Optimal neural population coding of an auditory spatial cue. Nature 430:682-686.

Jeffress LA (1948) A place theory of sound localization. J Comp Physiol Psychol 41:35-39.

Joris P, Yin TC (2007) A matter of time: internal delays in binaural processing. Trends Neurosci 30:70-78.

Joris PX, Van de Sande B, Louage DH, van der Heijden M (2006) Binaural and cochlear disparities. Proc Natl Acad Sci U S A 103:12917-12922.

Kapfer C, Seidl AH, Schweizer H, Grothe B (2002) Experience-dependent refinement of inhibitory inputs to auditory coincidence-detector neurons. Nat Neurosci 5:247-253.

Kuwabara N, Zook JM (1992) Projections to the medial superior olive from the medial and lateral nuclei of the trapezoid body in rodents and bats. J Comp Neurol 324:522-538.

Kuwada S, Stanford TR, Batra R (1987) Interaural phase-sensitive units in the inferior colliculus of the unanesthetized rabbit: effects of changing frequency. J Neurophysiol 57:1338-1360.

Kuwada S, Batra R, Stanford TR (1989) Monaural and binaural response properties of neurons in the inferior colliculus of the rabbit: effects of sodium pentobarbital. J Neurophysiol 61:269-282.

Lu T, Rubio ME, Trussell LO (2008) Glycinergic transmission shaped by the corelease of GABA in a mammalian auditory synapse. Neuron 57:524-535.

Magnusson AK, Kapfer C, Grothe B, Koch U (2005) Maturation of glycinergic inhibition in the gerbil medial superior olive after hearing onset. J Physiol 568:497-512.

Maki K, Furukawa S (2005) Acoustical cues for sound localization by the Mongolian gerbil, Meriones unguiculatus. J Acoust Soc Am 118:872-886.

Manley GA, Popper AN, Fay RR (2004) Evolution of the vertebrate auditory system. New York: Springer.

Mardia KV (1972) Statistics of directional data. London: Academic.
McAlpine D, Grothe B (2003) Sound localization and delay lines-do mammals fit the model? Trends Neurosci 26:347-350.

McAlpine D, Jiang D, Palmer AR (2001) A neural code for low-frequency sound localization in mammals. Nat Neurosci 4:396-401.

Moushegian G, Rupert AL, Gidda JS (1975) Functional characteristics of superior olivary neurons to binaural stimuli. J Neurophysiol 38:1037-1048.

Palmer AR (2004) Reassessing mechanisms of low-frequency sound localisation. Curr Opin Neurobiol 14:457-460.

Perkins RE (1973) An electron microscopic study of synaptic organization in the medial superior olive of normal and experimental chinchillas. J Comp Neurol 148:387-415.

Reed MC, Durbeck L (1995) Delay lines and auditory processing. Comm Theor Biol 3:441-461.

Schuller G, Radtke-Schuller S, Betz M (1986) A stereotaxic method for small animals using experimentally determined reference profiles. J Neurosci Methods 18:339-350.

Scott LL, Mathews PJ, Golding NL (2005) Posthearing developmental refinement of temporal processing in principal neurons of the medial superior olive. J Neurosci 25:7887-7895.

Scott LL, Hage TA, Golding NL (2007) Weak action potential backpropagation is associated with high frequency axonal firing capability in principal neurons of the gerbil medial superior olive. J Physiol 583:647-661.

Seidl AH, Grothe B (2005) Development of sound localization mechanisms in the mongolian gerbil is shaped by early acoustic experience. J Neurophysiol 94:1028-1036.

Shamma SA, Shen NM, Gopalaswamy P (1989) Stereausis: binaural processing without neural delays. J Acoust Soc Am 86:989-1006.

Siveke I, Pecka M, Seidl AH, Baudoux S, Grothe B (2006) Binaural response properties of low-frequency neurons in the gerbil dorsal nucleus of the lateral lemniscus. J Neurophysiol 96:1425-1440.

Siveke I, Leibold C, Grothe B (2007) Spectral composition of concurrent noise affects neuronal sensitivity to interaural time differences of tones in the dorsal nucleus of the lateral lemniscus. J Neurophysiol 98:2705-2715.

Smith AJ, Owens S, Forsythe ID (2000) Characterisation of inhibitory and excitatory postsynaptic currents of the rat medial superior olive. J Physiol 529:681-698.

Smith PH, Joris PX, Yin TC (1993) Projections of physiologically characterized spherical bushy cell axons from the cochlear nucleus of the cat: evidence for delay lines to the medial superior olive. J Comp Neurol 331:245-260.

Spirou GA, Rowland KC, Berrebi AS (1998) Ultrastructure of neurons and large synaptic terminals in the lateral nucleus of the trapezoid body of the cat. J Comp Neurol 398:257-272.

Spitzer MW, Semple MN (1995) Neurons sensitive to interaural phase disparity in gerbil superior olive: diverse monaural and temporal response properties. J Neurophysiol 73:1668-1690.

Ter-Mikaelian M, Sanes DH, Semple MN (2007) Transformation of temporal properties between auditory midbrain and cortex in the awake Mongolian gerbil. J Neurosci 27:6091-6102.

Tollin DJ, Yin TC (2005) Interaural phase and level difference sensitivity in lowfrequency neurons in the lateral superior olive. J Neurosci 25:10648-10657.

Turecek R, Trussell LO (2001) Presynaptic glycine receptors enhance transmitter release at a mammalian central synapse. Nature 411:587-590.

von Gersdorff H, Borst JG (2002) Short-term plasticity at the calyx of Held. Nat Rev Neurosci 3:53-64.

Wenthold RJ, Huie D, Altschuler RA, Reeks KA (1987) Glycine immunoreactivity localized in the cochlear nucleus and superior olivary complex. Neuroscience 22:897-912.

Yang L, Monsivais P, Rubel EW (1999) The superior olivary nucleus and its influence on nucleus laminaris: a source of inhibitory feedback for coincidence detection in the avian auditory brainstem. J Neurosci 19:2313-2325.

Yin TC, Chan JC (1990) Interaural time sensitivity in medial superior olive of cat. J Neurophysiol 64:465-488.

Yin TC, Kuwada S (1983) Binaural interaction in low-frequency neurons in inferior colliculus of the cat. II. Effects of changing rate and direction of interaural phase. J Neurophysiol 50:1000-1019.

Zhou Y, Carney LH, Colburn HS (2005) A model for interaural time difference sensitivity in the medial superior olive: interaction of excitatory and inhibitory synaptic inputs, channel dynamics, and cellular morphology. J Neurosci 25:3046-3058.

Zurek PM (1993) A note on onset effects in binaural hearing. J Acoust Soc Am 93:1200-1201. 